\title{
Comment on "Relativistic Aharonov-Bohm effect in the presence of planar Coulomb potentials"
}

\author{
C. R. Hagen * \\ Department of Physics and Astronomy \\ University of Rochester \\ Rochester, N.Y. 14627
}

\begin{abstract}
It is shown that the principal results of a recent work by Khalilov are incorrect. These errors are attributable to the author's insistence that wave functions must be regular at the origin even when the relevant potential is singular at that point.
\end{abstract}

PACS number(s): 03.65.Ge, 03.65.Nk, 03.65.Vf

In a recent paper Khalilov [1] has considered the problem of a charged spin one-half particle in combined AharonovBohm $(\mathrm{AB})$ and Coulomb potentials. A conspicuous feature of that work is the author's insistence that wave functions be regular at the origin. However, some years prior to that work the current author considered the spin one-half $\mathrm{AB}$ problem with one of the principal results being that wave functions singular at the origin played a crucial role in obtaining a consistent solution [2]. Subsequently the corresponding problem with a Coulomb potential included was considered in the Galilean or non-relativistic limit [3]. Again, it was found that singular solutions were required. Finally, the considerably more difficult problem of $\mathrm{AB}$ and Coulomb potentials together within the context of the Dirac equation (what might be called the ABCD problem) was investigated [4]. In this latter case it was found that there was no completely acceptable and physically reasonable solution of the ABCD problem.

Subsequent to [1] Khalilov [5] and Khalilov and Ho [6] have considered the same problem as that in ref. [2], again requiring the regularity of wave functions at the origin. This is particularly puzzling inasmuch as these authors were clearly aware [7] of the author's work in ref. [2]. It is the purpose of this brief note to comment briefly on these conflicting results and to note some of the obvious contradictions in refs. $[1,5,6]$.

The crucial error in [1] (and its sequels [5,6]) is to be found in Eq.(7) where the wave function is simply required to be regular, a considerably stronger requirement than that of mere normalizability. Since, however, the delta function term which governs the interaction of the spin with the magnetic field can be attractive, it should not be surprising to discover that for an approriate orientation of the spin relative to the magnetic field the wave function may need to be singular (but normalizable!). If the magnetized filament of the $\mathrm{AB}$ potential is taken to be of zero radius, the problem is highly singular and no obvious resolution is possible. This, however, is the approach of [1]. In [2] the filament is taken to have radius $R$ with the entire magnetic field confined to its surface (the extension to the case of an arbitrary radial distribution of the magnetic field within the region $r<R$ is considered in the Appendix). The required calculations have been carried out with some care in [2] with the intuitively reasonable result that the wave function requires the singular Bessel function in the single partial wave where both i) the interaction between the magnetized filament and the spin is attractive and ii) the wave function remains normalizable. This, of course, implies a spin dependent scattering amplitude and, consequently, the possibility of performing nontrivial polarization experiments.

In [1] it is found that the insistence on regular wave functions leads to spin independent scattering amplitudes. The author then proceeds to claim agreement with results of Alford and Wilczek [8]. However, ref.[8] considers only a repulsive spin interaction with the magnetic field, a case which can never lead to singular solutions. Had the author of [1] examined the subsequent work [9] of these authors, he would have found that they also found singular solutions in the attractive case, just as previously derived in [2].

It has already been noted that the spin dependence of the AB effect allows the detection of polarization effects. Since Khalilov finds no such spin dependence in the scattering amplitude, the assertion of the possibility of polarization effects in ref. [5] is manifestly inconsistent. In fact the polarization result which is claimed in [5] is obtained not from the (spin independent) scattering amplitude, but rather by an application of the usual spin rotation matrices. In order to display the correct polarization effects most clearly it is well to remark here that the calculation in [2] and that of [5] chose (rather unnaturally) a polarization direction which is the same for both the incident and scattered beams. In other words if the incident beam is polarized in a certain direction, then the detector accepts only those events which have the spin pointing in the same direction in space! The more general and more interesting case is that in which the detector selects events which do not make reference to the polarization of the incident beam. The results for the differential cross section for that case are [10] 


$$
\frac{d \sigma}{d \phi}=\left(\frac{d \sigma}{d \phi}\right)_{A B} \frac{1}{2}\left[1+(\mathbf{n} \cdot \hat{\mathbf{z}})\left(\mathbf{n}^{\prime} \cdot \mathbf{z}\right)-(\mathbf{n} \times \hat{\mathbf{z}})\left(\mathbf{n}^{\prime} \times \hat{\mathbf{z}}\right) \cos \phi-\hat{\mathbf{z}} \cdot\left(\mathbf{n} \times \mathbf{n}^{\prime}\right) \sin \phi\right]
$$

where $\left(\frac{d \sigma}{d \phi}\right)_{A B}$ is the usual AB differential cross section for an unpolarized beam. The vectors $\mathbf{n}$ and $\mathbf{n}^{\prime}$ denote respectively the polarization of the incident beam and the polarization of that part of the scattered beam which is accepted by the detector. It should be noted that this result is given for a beam which is incident from the right.

The above expression has two limits which are easily verified. First, in the case that $\mathbf{n}=\mathbf{n}^{\prime}$ it reduces to the result discussed in [2]. More interestingly, if one arranges that the detector only accepts events for which the orientation of the polarization vector $\mathbf{n}^{\prime}$ relative to the outgoing beam is identical to that of $\mathbf{n}$ with respect to the incident beam the differential cross section reduces [10] to the usual AB differential cross section. This is in agreement with the well known result that for a spin one-half particle in a magnetic field the component of the spin along the direction of propagation is conserved. It is furthermore a confirmation of the legitimacy of the procedure advanced in [2] for considering the AB effect as the limit of a magnetized filament of very small radius $R$.

[*] Electronic address: hagen@pas.rochester.edu

[1] V. R. Khalilov, Phys. Rev. A 71, 012105 (2005).

[2] C. R, Hagen, Phys. Rev. Lett. 64, 503 (1990).

[3] C. R. Hagen, Phys. Rev. D 48, 5935 (1993).

[4] C. R. Hagen and D. K. Park, Ann. Phys. (NY) 251, 45 (1996).

[5] V. R. Khalilov, Mod. Phys. lett. A 21. 1647 (2006).

[6] V. R. Khalilov and C-L Ho, e-print archive: hep-th/07083131 vl.

[7] See the acknowledgment in [5].

[8] M. G. Alford and F. Wilczek, Phys. Rev. Lett.62, 1071 (1989).

[9] M. G. Alford, J. March-Russell, and F. Wilczek Nucl Phys. B328, 140 (1989).

[10] C. R. Hagen, Phys. Rev. Lett. 64, 2347 (1990). 